\section{Treat periodontal diseases without antibiotics}

A friendly team of experts will be on Stand O22 waiting to show you the amazing benefits of PerioChip. With product demonstrations and practice-based applications to show you how to treat periodontal disease effectively without antibiotics, it's a must-see when you visit the BDIA Dental Showcase.

PerioChip is an easy to use, highly effective non-antibiotic adjunct treatment to reduce pocket depths in patients with adult periodontitis. After root surface debridement (RSD), PerioChip works with you to control bacteria, prevent infection and minimise the risk of tooth loss.

Designed specifically for periodontal pockets $\geq 5 \mathrm{~mm}$, this innovative, biodegradable insert optimises treatment outcomes by eliminating $99 \%$ of subgingival pathogenic bacteria over seven to ten days after placement. As well as this, the antimicrobial effects of PerioChip carry on working to suppress bacterial growth for up to 11 weeks, allowing effective healing and stabilisation.

Clinical studies into the efficacy of PerioChip show that combining RSD with PerioChip can offer five times more effective results in the reduction of periodontal pockets versus RSD alone.

When you have PerioChip at your side, you have a product you can count on. There is no wonder it's been described as a 'first line treatment' for patients with periodontal disease.

To order PerioChip or for further information call free on 0800013 2333 or email team@periochip.co.uk.

\section{Win a staycation for two}

As one of the world's foremost manufacturers of high-quality dental technology, A-dec has something for everyone - and will be showcasing its extensive and widely popular range of dental chairs, stools, lights and cabinets to delegates.

This, of course, includes products such as the premier A-dec 500, which has been designed for optimal ergonomics and patient access, or the newly updated A-dec 300, with unprecedented modular flexibility and adaptability.

What's more, the friendly A-dec team will be encouraging delegates to try designing their next A-dec chair using the new my A-dec App an innovative programme that allows practitioners to design the chair they need, to their own preferences and clinical specifications. All delegates that take advantage of this excellent opportunity will also be entered into a draw to win a short break

for two in the UK.

Truly, this is an unmissable chance to talk to the A-dec team and learn more about the wide range of dental solutions, so make sure you save the time to visit stand I20 at BDIA Dental Showcase 2016

For more information about A-dec Dental UK Ltd, visit www.a-dec.co.uk or call on 0800233285.

\title{
Take a journey through a quarter century of clinical advances
}

This year Philips will be taking Showcase delegates on a voyage of discovery. The journey starts as they enter the Philips stand and will guide them through a quarter century of clinical advances which has ch anged the face of oral healthcare and tooth whitening. The journey also encompasses the breadth of the portfolio from tooth brushing, interdental and tongue cleaning technology through to tooth whitening.

Philips will also be signposting several new product launches and allowing delegates to get their hands on the products so that they can explore every facet for themselves with the help of their travel guides - the TBM team.

At the start of the Philips journey is the ubiquitous Sonicare - the innovation which initiated a whole new dental sector for sonic tooth cleaning and has become the sonic toothbrush most dental professionals worldwide recommend for good reason. Patients can choose from the classic EasyClean or the designer DiamondClean.

2016 sees the launch of a new fashion-forward colour choice for the DiamondClean ensuring this multi award winning brush continues to go from strength to strength this year. 2016 also saw the launch of Sonicare for Kids Connected, and Showcase will see the Sonicare FlexCare Platinum take adults to an exciting new level of connectivity.

Sonicare brushes are even better when used with the pioneering AirFloss Pro interproximal cleaner, which sets the gold standard for exemplary oral care and improves gum health in two weeks. This, when coupled with TongueCare + , cleans areas of the mouth even devices cannot reach for the ultimate in fresh breath.
Philips Zoom is the number one patient-requested professional whitening treatment. It comprises Zoom WhiteSpeed - the only in-practice light-activated system with variable intensity settings to maximise patient comfort and a gel which includes amorphous calcium phosphate (ACP) to further minimise sensitivity and protect tooth enamel. This is best combined with Product of the Year Winner, Philips Zoom NiteWhite and DayWhite at-home whitening for a visibly whiter smile in 1-2 weeks. The newest introduction to the tooth whitening portfolio is Zoom QuickPro with its paint on, brush off convenience, and four shade results in four days. A new Zoom whitening initiative will also be unveiled at the show.

Visit stand N40 at BDIA Showcase or www.philips-tsp.co.uk/sonicare. 